\title{
Joint Power-Delay Minimization in Green Wireless Access Networks
}

\author{
Farah Moety*, Samer Lahoud*, Kinda Khawam ${ }^{\dagger}$, Bernard Cousin* \\ *University of Rennes I - IRISA, France \\ ${ }^{\dagger}$ University of Versailles - PRISM, France
}

\begin{abstract}
The growing energy demands, the increasing depletion of traditional energy resources, together coupled with the recent explosion of mobile internet traffic call for green solutions to address the challenges in energy efficient wireless access networks. In this paper, we consider possible power saving through reducing the number of active BSs and adjusting the transmit power of those that remain active while maintaining a satisfying service for all users in the network. Thus, we introduce an optimization problem that jointly minimizes the power consumption of the network and the sum of the transmission delays of the users in the network. Our formulation allows investigating the tradeoff between power and delay by tuning the weighting factors associated to each one. Moreover, to reduce the computational complexity of the optimal solution of our nonlinear optimization problem, we convert it into a Mixed Integer Linear Programming (MILP). We provide extensive simulations for various decision preferences such as power minimization, delay minimization and joint minimization of power and delay. Presented results show that we obtain power savings up to $20 \%$ compared to legacy network models.
\end{abstract}

\section{INTRODUCTION}

Pushed by the needs to reduce energy, mobile operators are rethinking their network design for optimizing its energy efficiency and satisfying user Quality of Service (QoS) requirement

Currently, over $80 \%$ of the power in mobile telecommunications is consumed in the radio access network, more specifically at the base stations (BSs) level [10]. Thus, most of studies on energy efficiency in wireless network focus on the radio access. In the literature, various techniques are proposed for improving energy efficiency in wireless access networks such as decreasing the cell size, adapting power consumption to traffic load [3] or devising intelligent network deployment strategies using small, low power, femtocells and relays [10][12]. However satisfying user QoS has not been considered as a constraint in these works. Notable exceptions are the works in [8] and [11]. In [8], the authors proposed an optimization approach that minimizes power consumption in wireless access networks while ensuring coverage of active users and enough capacity for guaranteeing QoS. In [11], the authors formulated a cost minimization problem that allows for a flexible tradeoff between flow-level performance and energy consumption.

In this paper, we tackle the problem of power saving while minimizing the user delay in wireless access networks by finding a tradeoff between reducing the number of active BSs and adjusting the transmit power of those that remain active while maintaining a satisfying service for all users in the network. Thus, we formulate an optimization problem that jointly minimizes the power consumption of the network and the sum of data unit transmission delays of all users in the network. Compared to prior works in the state of the art taking into consideration power saving and QoS, the optimization approach proposed in [8] does not support the feature of tuning the weights associated to the power and QoS cost; [11] uses an M/GI/1 queue for the delay model, which is a pessimistic bound compared to the realistic delay model we use in our paper. Moreover, [11] studies only the case where BSs switch between on and off modes without adjusting their transmit power.

We also tackle in this work the problem of user association: when an active BS is switched off or changes its transmit power level, users may need to change their associations. This coupling makes the problem more challenging. In our formulation, we consider the case of a Wireless Local Area Network (WLAN) using IEEE 802.11g technology.

- The running mode (on/off) of the network BSs and for active BSs, the corresponding transmit power level.

- The association of each user to which BS.

The key contributions of our work are as follows:

- We formulate the problem of power-delay minimization in wireless access networks, going beyond the prior work in the literature which has focused either on minimizing energy without taking into consideration the QoS (i.e., delay) [10]-[3]-[12] or on delay analysis without taking into account energy minimization [9]-[7].

- Our problem formulation is multiobjective. Thus, it allows investigating the power-delay tradeoffs by tuning the weights associated to the total network cost components, namely power and delay.

- Starting from a non-linear formulation of the problem, we provide a linearization process that makes the problem computationally tractable for realistic scenarios.

- The delay model provided in this paper is a unique feature of our work, it is the realistic model used in IEEE 802.11 WLAN [6]-[9]-[7].

The rest of the paper is organized as follows. In Section II, we describe the system model considering an IEEE 802.11 WLAN. In Section III we present our proposed optimization approach and the linearization process. In Section IV we provide extensive simulation results. Conclusions and perspectives are given in Section $\mathrm{V}$. 


\section{Nework Model}

We consider the energy consumption of IEEE $802.11 \mathrm{~g}$ WLAN, with Access Points (APs) working in infrastructure mode. We refer in the following to the term BS by the term AP as we consider the case of WLANs. As the downlink traffic on mobile networks is still today several orders higher than the uplink traffic, we only consider the downlink traffic (e.g., accessing web data) sent from AP to users. We assume that the network is in a static state where users are stationary. In other words, we take a snapshot of a dynamic system and optimize its current state. Furthermore, we assume that the network is in a saturation state, which means that we treat a worst case scenario where every user has persistent traffic. Moreover, when the AP is switched on, it is able to transmit at different power levels. We denote by $N_{a p}$ and $N_{l}$ the number of APs in the network and the number of transmit power levels respectively. The indexes $i \in I=1, \ldots, N_{a p}$, and $j \in J=$ $1, \ldots, N_{l}$, are used throughout the paper to designate a given $\mathrm{AP}$ and its transmit power level respectively. Note that, for $j=1$ we consider that the AP transmits at the highest power level and for $j=N_{l}$ the AP is switched off. We term by $k \in K=1, \ldots, N_{u}$, the index of a given user where $N_{u}$ is the number of users in the network.

\section{A. Power Consumption Model}

Adopting the proposed model in [10], the power consumption of an AP is modeled as a linear function of average transmit power per site as below:

$$
p_{i, j}=L \cdot\left(a \pi_{j}+b\right)
$$

where $p_{i, j}$ and $\pi_{j}$ denote the average consumed power per AP $i$ and the transmit power at level $j$ respectively. The coefficient $a$ accounts for the power consumption that scales with the transmit power due to radio frequency amplifier and feeder losses while $b$ models the power consumed independently of the transmit power due to signal processing and site cooling. $\mathrm{L}$ reflects the activity level of the APs. As we assume that the network is in a saturation state, $\mathrm{L}$ is equal to one, e.g., each active AP has at least one mobile requesting data with all resources allocated.

The cell coverage area in a cellular system is defined as the expected percentage of area within a cell that receives power above a given minimum. The transmit power at the AP is designed for an average received power at the cell boundary [4]. Thus, transmitting at different power levels leads to different coverage area sizes. Note that, all users within a cell require some minimum received Signal to Noise Ratio (SNR) for acceptable performance. Thus, in our paper, a user is considered covered by an AP if his SNR is above a given threshold.

\section{B. Delay Model}

We adopt the delay model presented in [6] and validated in our previous works [9]-[7]. We denote by $\chi_{i, j, k}$ the peak rate perceived by user $k$ from AP $i$ transmitting at level $j$. Thus, when user $k$ is associated to AP $i$ transmitting at level $j$, his perceived data rate is given by [7]: $R_{i, j, k}=$ $1 / \frac{1}{\chi_{i, j, k}}+\sum_{k^{\prime}=1, k^{\prime} \neq k}^{N_{u}} \frac{\theta_{i, k^{\prime}}}{\chi_{i, j, k^{\prime}}}$, where $\theta_{i, k^{\prime}}$ is a binary variable that indicates whether user $k^{\prime}$ is associated to $\mathrm{AP} i$ or not. We denote by $T_{i, j, k}$ the amount of time necessary to send a data unit to user $k$ from AP $i$ transmitting at level $j$. In fact, the delay needed to transmit a bit for a given user is the inverse of the throughput perceived by this user. Thus,

$$
T_{i, j, k}=1 / R_{i, j, k}=\frac{1}{\chi_{i, j, k}}+\sum_{k^{\prime}=1, k^{\prime} \neq k}^{N_{u}} \frac{\theta_{i, k^{\prime}}}{\chi_{i, j, k^{\prime}}} .
$$

Note that this model can be easily adapted to other wireless technologies such as 2G, WiMAX, or LTE systems [7].

\section{OPtimization PROBlem}

\section{A. Problem Formulation}

Our approach can be formulated as an optimization problem $(\mathcal{P})$ that consists in minimizing the power consumption of the network and the sum of the data unit transmission delays of all active users. These two minimization objectives are conflicting objectives since the bit transmission delay decreases as the transmit power of the AP increases. Thus, a joint optimization of power and delay enables to tune the impact of each one.

We define the total network power and the total network delay as follows: the total network power is defined as the total power consumption of active APs in the network. The power consumption of an active AP, given in Equation 1, is modeled as a linear function of the average transmit power. Let $\lambda_{i, j}$ be a binary variable that indicates whether AP $i$ transmits at level $j$ or not. Thus, the total network power, denoted by $C_{p}\left(\lambda_{i, j}\right)$, is given by:

$$
\begin{array}{r}
C_{p}\left(\lambda_{i, j}\right)=\sum_{i \in I} p_{i}\left(\lambda_{i, j}\right)=\sum_{i \in I, j \in J} p_{i, j} \cdot \lambda_{i, j} \\
=\sum_{i \in I, j \in J}\left(a \pi_{j}+b\right) \cdot \lambda_{i, j}
\end{array}
$$

The total network delay is defined as the sum of data unit transmission delays of all users in the network. The data unit transmission delay $T_{i, j, k}$ of user $k$ associated to AP $i$ transmitting at level $j$ is given in Equation 2. This delay depends on the transmit power of the AP the user is associated to. Recall that the binary variable $\theta_{i, k}$ indicates whether a user $k$ is associated to AP $i$ or not. Thus, the total network delay, denoted by $C_{d}\left(\theta_{i, k}, \lambda_{i, j}\right)$, is given by:

$$
\begin{array}{r}
C_{d}\left(\lambda_{i, j}, \theta_{i, k}\right)=\sum_{i \in I, k \in K} T_{k}\left(\lambda_{i, j}\right) \cdot \theta_{i, k} \\
=\sum_{i \in I, j \in J, k \in K} T_{i, j, k} \cdot \lambda_{i, j} \cdot \theta_{i, k}
\end{array}
$$

Consequently, the total network cost, denoted by $C_{t}\left(\theta_{i, k}, \lambda_{i, j}\right)$, is defined as the weighted sum of power and delay components and is given by the following:

$$
C_{t}\left(\lambda_{i, j}, \theta_{i, k}\right)=\alpha C_{p}\left(\lambda_{i, j}\right)+\beta \beta^{\prime} C_{d}\left(\theta_{i, k}, \lambda_{i, j}\right)
$$

$\beta^{\prime}$ is a normalization factor and $\alpha$ and $\beta$ are the weighting factors that tune the tradeoff between the two components of 
the total network cost. Note that $\alpha+\beta=1$ and that $\alpha$ and $\beta \in[0,1]$. In particular, when $\alpha$ equals 1 and $\beta$ equals 0 , we only focus on the power saving, and as $\alpha$ decreases and $\beta$ increases more emphasis is put on the delay component.

Our problem $(\mathcal{P})$ consists in finding an optimal set of active APs transmitting at a specific power level and an optimal user association that minimize the total network $\operatorname{cost} C_{t}\left(\lambda_{i, j}, \theta_{i, k}\right)$. Therefore $(\mathcal{P})$ is given by:

$$
\begin{aligned}
& \text { Min } C_{t}\left(\lambda_{i, j}, \theta_{i, k}\right)=\alpha \sum_{i \in I, j \in J}\left(a \pi_{j}+b\right) \cdot \lambda_{i, j} \\
& +\beta \beta^{\prime} \sum_{i \in I, j \in J, k \in K}\left(\frac{\lambda_{i, j} \cdot \theta_{i, k}}{\chi_{i, j, k}}+\sum_{k^{\prime} \in K, k^{\prime} \neq k} \frac{\lambda_{i, j} \cdot \theta_{i, k} \cdot \theta_{i, k^{\prime}}}{\chi_{i, j, k^{\prime}}}\right)
\end{aligned}
$$

Subject to:

$$
\begin{gathered}
\sum_{j \in J} \lambda_{i, j}=1 \quad \forall i \in I \\
\sum_{i \in I} \theta_{i, k}=1 \quad \forall k \in K \\
\lambda_{i, j} \cdot \theta_{i, k}=0 \quad \forall(i, j, k): i \in I, j=N_{l}, k \in K \\
\lambda_{i, j} \in\{0,1\} \quad \forall(i, j): i \in I, j \in J \\
\theta_{i, k} \in\{0,1\} \quad \forall(i, k): i \in I, k \in K
\end{gathered}
$$

The objective function (6) provides the total cost of the network in terms of power and delay. Constraints (7) ensure that a given user is connected to only one AP. Constraints (8) state that every AP transmits only at one power level. In practice, when turning off some APs to accomplish power saving, some users will be uncovered. Thus, in our problem, to prevent users to be associated to a switched off AP, we add constraints (9). These equations ensure that $\lambda_{i, N_{l}}$ and $\theta_{i, k}$ are not both equal one. Indeed, when AP $i$ is switched off, $\lambda_{i, N_{l}}$ is equal to one, then $\theta_{i, k}$ of all users cannot be equal to one. Constraints (10) and (11) are the integrality constraints for the decision variables $\lambda_{i, j}$ and $\theta_{i, k}$.

Moreover, to eliminate some trivial cases that are not included in the solution, we add the following constraints:

- If user $k$ is not covered by AP $i$ transmitting at the first (highest) power level, then:

$$
\theta_{i, k}=0
$$

The equalities (12) prevent a given user to be associated to an AP if that user is not in the AP first power level coverage area.

- If user $k$ is not covered by AP $i$ transmitting at power level $j, j \in\left\{2, . ., N_{l}-1\right\}$, then:

$$
\lambda_{i, j} \cdot \theta_{i, k}=0 \quad \forall j \in\left\{2, . ., N_{l}-1\right\}
$$

The equalities (13) ensure that $\lambda_{i, j}$ and $\theta_{i, k}$ are not both equal one, and this prevents a given user to be associated to an AP if that user is not in the AP $j^{\text {th }}$ power level coverage area.

Hence, solving problem $(\mathcal{P})$ gives:

- The running mode of each AP and its corresponding transmit power. This is designated by $\lambda_{i, j}$ for each $i \in I$ and $j \in J$.
- The users association designated by $\theta_{i, k}$ for each $i \in I$ and $k \in K$.

\section{B. Linearization Process}

To reduce the complexity of our non-linear optimization problem $(\mathcal{P})$, we convert it into a Mixed Integer Linear Programming (MILP). Thus, we replace the non-linear terms by new variables and additional inequality constraints, which ensure that new variables behave according to the non-linear terms they are replacing. Particularly, in the objective function (Equation 6) and in the constraints (9) and (13), we replace each quadratic term $\lambda_{i, j} \cdot \theta_{i, k}$ by a new linear variable $y_{i, j, k}$ and add the following three inequalities to the set of constraints:

$$
\begin{gathered}
y_{i, j, k}-\lambda_{i, j} \leq 0 \quad \forall(i, j, k): i \in I, j \in J, k \in K \\
y_{i, j, k}-\theta_{i, k} \leq 0 \quad \forall(i, j, k): i \in I, j \in J, k \in K \\
\lambda_{i, j}+\theta_{i, k}-y_{i, j, k} \leq 1 \quad \forall(i, j, k): i \in I, j \in J, k \in K
\end{gathered}
$$

The inequalities (14) and (15) ensure that $y_{i, j, k}$ equals zero when either $\lambda_{i, j}$ or $\theta_{i, k}$ equals zero, while the inequalities (16) force $y_{i, j, k}$ to be equal to one if both $\lambda_{i, j}$ and $\theta_{i, k}$ equal one.

Similarly, we replace in the objective function (Equation 6) each quadratic term $\lambda_{i, j} \cdot \theta_{i, k} \cdot \theta_{i, k^{\prime}}$ by a new variable $z_{i, j, k, k^{\prime}}$ and add the following inequalities to the set of constraints:

$$
\begin{array}{r}
z_{i, j, k, k^{\prime}}-\lambda_{i, j} \leq 0 \quad \forall\left(i, j, k, k^{\prime}\right): i \in I, j \in J, k<k^{\prime} \in \underset{(17)}{K} \\
z_{i, j, k, k^{\prime}}-\theta_{i, k} \leq 0 \quad \forall\left(i, j, k, k^{\prime}\right): i \in I, j \in J, k<k^{\prime} \in \underset{(18)}{K} \\
z_{i, j, k, k^{\prime}}-\theta_{i, k^{\prime}} \leq 0 \quad \forall\left(i, j, k, k^{\prime}\right): i \in I, j \in J, k<k^{\prime} \in \underset{(19)}{K} \\
\lambda_{i, j}+\theta_{i, k}+\theta_{i, k^{\prime}}-z_{i, j, k, k^{\prime}} \leq 2 \quad \forall\left(i, j, k, k^{\prime}\right): i \in I, j \in J, \\
k<k^{\prime} \in K \\
z_{i, j, k, k^{\prime}}-z_{i, j, k^{\prime}, k}=0 \quad \forall\left(i, j, k, k^{\prime}\right): i \in I, j \in J, k<k^{\prime} \in K
\end{array}
$$

The inequalities (17), (18) and (19) ensure that $z_{i, j, k, k^{\prime}}$ is equal to zero when either $\lambda_{i, j}$ or $\theta_{i, k}$ or $\theta_{i, k^{\prime}}$ equals zero, while the inequalities (20) force $y_{i, j, k}$ to be equal to one if $\lambda_{i, j}, \theta_{i, k}$ and $\theta_{i, k^{\prime}}$ are equal to one. Furthermore, as $\lambda_{i, j} \cdot \theta_{i, k} \cdot \theta_{i, k^{\prime}}=$ $\lambda_{i, j} \cdot \theta_{i, k^{\prime}} \cdot \theta_{i, k}$, constraints (21) force $z_{i, j, k, k^{\prime}}$ to be equal to $z_{i, j, k^{\prime}, k}$.

In addition, we give the bound constraints for the variables $y_{i, j, k}$ and $z_{i, j, k, k^{\prime}}$ which are introduced during the linearization process:

$$
\begin{gathered}
0 \leq y_{i, j, k} \leq 1 \quad \forall(i, j, k): i \in I, j \in J, k \in K \\
0 \leq z_{i, j, k, k^{\prime}} \leq 1 \quad \forall\left(i, j, k, k^{\prime}\right): i \in I, j \in J, k<k^{\prime} \in K
\end{gathered}
$$

Finally, our MILP problem $\left(\mathcal{P}^{\prime}\right)$ is given by:

$$
\begin{array}{r}
\text { Min } C_{t}\left(\lambda_{i, j}, y_{i, j, k}, z_{i, j, k, k^{\prime}}\right)=\alpha \sum_{i \in I, j \in J}\left(a \pi_{j}+b\right) \cdot \lambda_{i, j} \\
+\beta \beta^{\prime} \sum_{i \in I, j \in J, k \in K}\left(\frac{y_{i, j, k}}{\chi_{i, j, k}}+\sum_{k^{\prime} \in K, k^{\prime} \neq k} \frac{z_{i, j, k, k^{\prime}}}{\chi_{i, j, k^{\prime}}}\right)
\end{array}
$$




\begin{tabular}{|l|l|}
\hline Notation & Definition \\
\hline$N_{a p}$ & The number of APs \\
\hline$N_{l}$ & The number of transmit power levels \\
\hline$N_{u}$ & The number of users \\
\hline$p_{i, j}$ & $\begin{array}{l}\text { The average consumed power per AP } i \\
\text { transmitting at power level } j\end{array}$ \\
\hline$\pi_{j}$ & The transmit power at level $j$ \\
\hline$\chi_{i, j, k}$ & $\begin{array}{l}\text { The peak rate perceived by user } k \\
\text { from AP } i \text { transmitting at level } j\end{array}$ \\
\hline$T_{i, j, k}$ & $\begin{array}{l}\text { The amount of time necessary to send a data unit to user } k \\
\text { from AP } i \text { transmitting at level } j\end{array}$ \\
\hline$\theta_{i, k}$ & $\begin{array}{l}\text { A binary variable that indicates whether user } k \\
\text { is connected to AP } i\end{array}$ \\
\hline$\lambda_{i, j}$ & $\begin{array}{l}\text { A binary variable that indicates whether an AP } i \\
\text { transmits at power level } j\end{array}$ \\
\hline$y_{i, j, k}$ & $\begin{array}{l}\text { A binary variable that indicates whether user } k \text { is associated } \\
\text { to AP } i \text { transmitting at power level } j\end{array}$ \\
\hline$z_{i, j, k, k^{\prime}}$ & $\begin{array}{l}\text { A binary variable that indicates whether user } k \text { and user } k^{\prime} \\
\text { are associated to AP } i \text { transmitting at power level } j\end{array}$ \\
\hline
\end{tabular}

Table I

NOTATION SUMMARY

Subject to the constraints:

(7)-(23).

The main notations used in the present paper are reported in Table I.

\section{Performance Evaluation}

\section{A. Evaluation Methodology}

To evaluate the tradeoff between power and delay, we compute the optimal solution of our ILP using GLPK (GNU Linear Programming Kit) solver over a network topology composed of twelve cells $\left(N_{a p}=12\right)$ covered by IEEE $802.11 \mathrm{~g}$ technology and six users in each cell $\left(N_{u}=12 * 6=72\right)$. The positioning of the WLAN APs in the network is performed following a grid structure and the positioning of users is generated randomly following a uniform distribution.

For the APs power model, we set for simplicity the number of transmit power levels to three $\left(N_{l}=3\right)$. Indeed, in this paper, we aim at computing the optimal solution of the MILP problem thus if we increase $N_{l}$, the granularity will be finer but the problem will be intractable. We note that when $j=N_{l}=3$, the AP is switched off, whereas an active AP is able to transmit at two different power levels. The input parameters of the power consumption model in Equation 1 are given below:

- $a=3.2, b=10.2$ [12]

- $\pi_{1}=0.03 \mathrm{~W}$ and $\pi_{2}=0.015 \mathrm{~W}$ [2] are the transmit powers when the AP is running on the first and the second power levels respectively.

Hence, the average consumed power per AP $i$ at the first and the second power levels are given respectively by $p_{i, 1}=$ $10.296 \mathrm{~W}$ and $p_{i, 2}=10.248 \mathrm{~W}(i=1, \ldots, 72)$. As mentioned earlier, we assume that for $j=N_{l}=3$, the AP is switched off and thereby $p_{i, 3}=0$.

In addition, the coverage radius for the first and the second power levels are respectively $R_{1}=107,4 \mathrm{~m}$ and $R_{2}=75,8 \mathrm{~m}$. We obtain these values by simulation on Network Simulator (NS2) for a SNR threshold equals to $-0.5 \mathrm{~dB}$ at the cell boundary. This SNR is the minimum value to be maintained in order to consider that a given user is covered by the AP. It corresponds to a cell boundary peak rate that equals $1 \mathrm{Mb} / \mathrm{s}$ in the downlink. Precisely, we implement in NS2 a benchmark scenario consisting of a free propagation model to characterize the WLAN radio environment, an IEEE $8021.11 \mathrm{~g}$ AP working on $2.462 \mathrm{GHz}$ (channel 11) and a single user at different positions. This user is receiving from the AP a Constant Bit Rate (CBR) traffic with a packet size of 1000 bytes and an inter-arrival time of $0.4 \mathrm{~ms}$ corresponding to a rate of $20 \mathrm{Mb} / \mathrm{s}$. This leads to saturation state of the network according to the assumption presented in section II. In these conditions, the throughput experienced by our single user is the maximum achievable throughput (peak rate) for the current SNR. As changes in transmit power influence the radio channel conditions, we run this scenario for each transmit power level of the AP $\left(\pi_{1}=0.03 \mathrm{~W}\right.$ and $\left.\pi_{2}=0.015 \mathrm{~W}\right)$ to obtain respectively $\chi_{i, 1, k}$ and $\chi_{i, 2, k}$ for the corresponding user. When the average peak rate of the user is equal to $1 \mathrm{Mb} / \mathrm{s}$ (target peak rate at the cell edge), we note the distance between the user and the AP for the two power levels and thus we obtain the corresponding radius $R_{1}$ and $R_{2}$. Figure 1 shows the peak rate perceived by the user from the AP, transmitting at the first and the second power level, as a function of the distance between them.

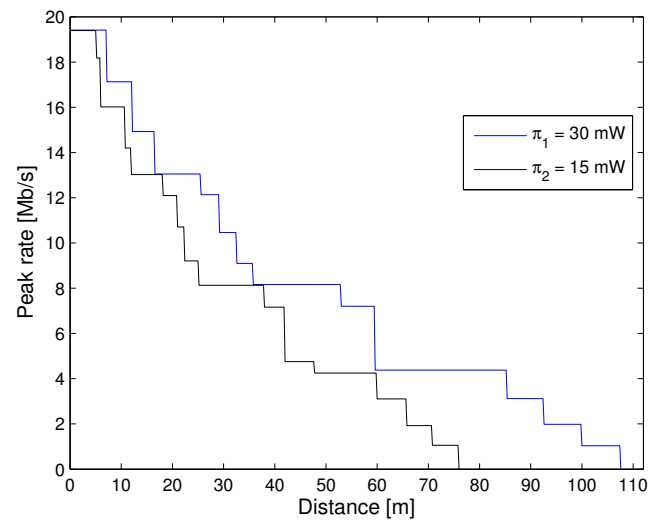

Figure 1. Peak rates in IEEE 802.11g for different transmit power levels.

To ensure the wireless signal reception by all users in the network, we generate their positioning in such a way each user is covered by at least one AP when all APs transmit at the highest power level. Figure 2 shows an example of

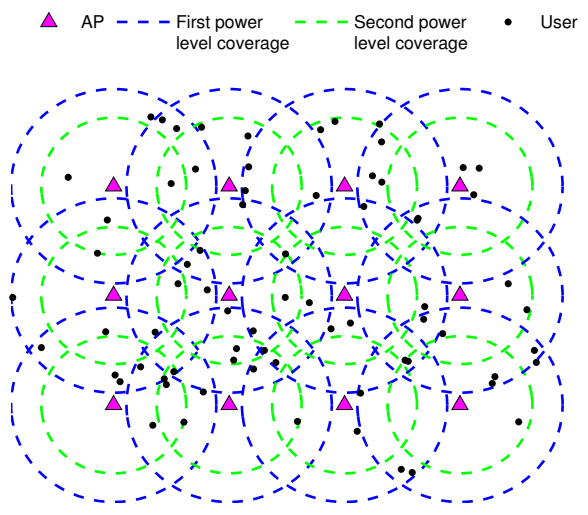

Figure 2. Network topology with inter-cell distance $D=120.8 \mathrm{~m}$. 


\begin{tabular}{|c|c|c|c|c|c|c|c|c|}
\hline Inter-cell distance $[\mathrm{m}]$ & 120.8 & 134.2 & 147.6 & 161.1 & 174.5 & 187.9 & 201.3 & 214.8 \\
\hline Average number of wireless signal layers & 2.12 & 1.83 & 1.58 & 1.40 & 1.26 & 1.15 & 1.05 & 1.00 \\
\hline
\end{tabular}

Table II

WIRELESS SIGNAL LAYERS VS INTER-CELL DISTANCE.

our network topology where the distance between the APs is $120.8 \mathrm{~m}$. With this distance, we obtain a dense coverage area where the average number of wireless signal layers is 2.12 (Table II). Table II shows the average number of wireless signal layers as a function of the distance $(D)$ between the APs. In other words, it reflects, for each user, the average of number of covering APs (transmittting at the highest power level) with different inter-cell distances. $D$ varies from $\left(R_{1}+\right.$ $\left.1 / 8 R_{1}=120.8 \mathrm{~m}\right)$ to $\left(2 R_{1}=214.8 \mathrm{~m}\right)$ with a step of $1 / 8 R_{1}$ $=13.425 \mathrm{~m}$. As $D$ increases, the average number of wireless signal layers decreases as low as one when there is no overlap between the cells $\left(D=2 R_{1}\right)$.

Toward studying the tradeoff between minimizing the power consumption of the network and minimizing the sum of users delay in the network, we tune the values of the weights $\alpha$ and $\beta$ associated to power and delay components respectively, and investigate the obtained solutions. We consider three settings: the two weights are equal, $\alpha$ is very large compared to $\beta$ and $\beta$ is very large compared to $\alpha$. Practically, the first setting, named Power-Delay-Min, matches the case where the power and delay components of the total network cost are equally important. The second setting, named Power-Min, matches the case where more preference is given to power saving. On the opposite, the third setting, named Delay-Min, matches the case where more importance is given to minimizing the delay component.

We compare the performance of our MILP solution for the considered settings with reference models for power and user association. The reference power model is denoted by the Highest Power Level (HPL) model as it assumes that all the APs transmit at the highest power level $(j=1)$. Under these circumstances, we consider a Power-Based user association (PB-UA) model. PB-UA model takes into consideration the power of the received signal at the user side in such a way the user connects to the AP where it gets the highest SNR. Afterward, the delay is calculated using Equation 2 according to the PB-UA model. These reference models are similar to the most frequently deployed WLAN networks, where APs transmit at a fixed transmit power level and users connect to the AP where they get the highest received signal strength [1]. Note that all results are the mean over 50 simulations with $95 \%$ confidence interval. Moreover, the normalization factor $\beta^{\prime}$ is calculated in each simulation in such a way to scale the two components of the total network cost [5].

\section{B. Simulation Results}

We start by examining how much power saving can be achieved for the three considered cases with respect to the HPL model while varying the inter-cell distance $(D)$. The power saving is defined as: 1-the ratio between the total network power for the considered case and the total network power for the HPL model. Note that the total network power, defined

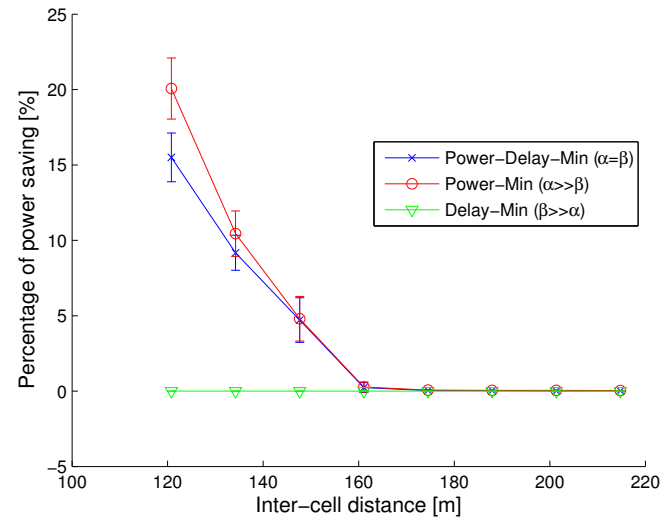

Figure 3. Power Saving for the three considered cases with respect to HPL model.

in Equation 3 for the HPL model where all APs transmit at the first power level, equals $\sum_{i=1}^{12} P_{i, 1}=12 * 10.296=$ $123.552 \mathrm{~W}$. Figure 3 plots the percentage of power saving for the three considered cases as a function of the intercell distance. The Power-Min and the Power-Delay-Min cases show decreasing curves for $D$ ranging between $120.8 \mathrm{~m}$ to $161.1 \mathrm{~m}$. Furthermore, they have no power saving gain for $D$ $\geq 161.1 \mathrm{~m}$. As expected, the figure shows that the Power-Min case has the highest percentage of power saving at $20 \%$ for $D=120.8 \mathrm{~m}$, followed by the Power-Delay-Min case at $15 \%$ for the same $D$. The Delay-Min case has no power saving gain for all distances. In other words, in the Delay-Min case, we obtain a network configuration similar to the HPL model where all the APs transmit at the highest power level. This is because in this case we are interested in minimizing the sum of the users delay, therefore when all APs transmit at the highest level, users will experience higher throughput in comparison with the case where some of the APs transmit at the second power level or are switched off.

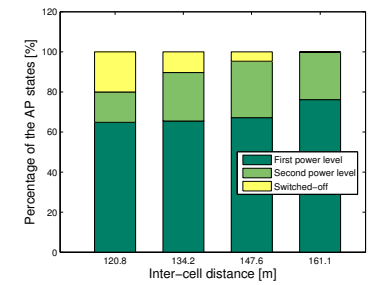

(a) Power-Min $(\alpha \gg \beta)$

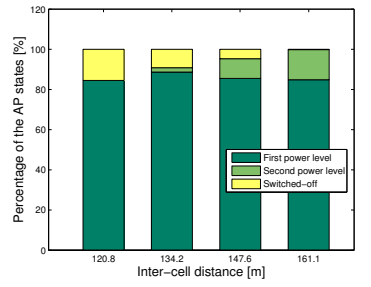

(b) Power-Delay-Min $(\alpha=\beta)$ Figure 4. Percentage of the AP states for Power-Min and Power-Delay-Min cases (a) (b).

In order to examine the origin of power savings in the Power-Min and Power-Delay-Min cases, we plot Figures 4(a) and 4(b) that illustrate the percentage of the APs state for $D$ equals $120.8 \mathrm{~m}$ to $161.1 \mathrm{~m}$. We see that in the PowerMin case, we obtain percentages of APs transmitting at the second power level and switched off greater than that in the Power-Delay-Min case for the different values of $D$. 
Moreover, for the two cases, we see that when $D$ increases the percentage of switched off APs decreases, and the percentage of APs transmitting at the second power level increases. On the one hand, this explains the decreasing curves for the corresponding inter-cell distances in Figure 3. On the other hand, this behaviour is due, for low values of $D$, to the relatively high number of wireless signal layers; thus, the possibility to switch off the AP or to transmit at low power level is high. While when $D$ increases, the number of wireless signal layers decreases and thus the possibility to switch off the AP or to transmit at low power level decreases in order to ensure coverage for all users in the network.

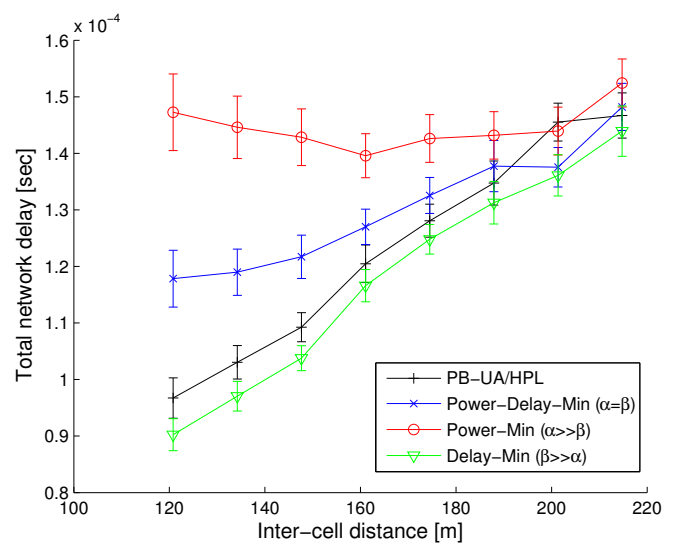

Figure 5. Total network delay for the three considered cases and for PBUA/HPL model.

We now investigate the total network delay for the considered cases compared to the PB-UA/HPL model while varying the inter-cell distance $(D)$. As expected, for the comparison of the three cases, Figure 5 shows that the Delay-Min case has the lowest delay cost, followed by the Power-Delay-Min case and finally by the Power-Min case. Moreover, the figure shows that the curve corresponding to the PB-UA with the HPL model falls below the curves corresponding to the PowerDelay-Min and the Power-Min cases and performs close to the optimal solution of the Delay-Min case. This is because in the Delay-Min case, we obtain a network configuration where all APs transmit at the highest power level (similar to the HPL model) and thus the problem becomes a user association problem that aims to minimize the sum of delays of all users in the system. Further, we see that the delay cost of the Delay-Min has an increasing curve. This is because, for the same users distribution, when $D$ increases, the received power at the user side will decrease and thus the perceived rate at the current SNR will decrease which will cause the delay to increase. For the same reason, we see also that the Power-Delay-Min case has an increasing curve but with lower slope at the first inter-cell distances. While, the Power-Min curve shows a decreasing curve for $D$ between $120.8 \mathrm{~m}$ and $161.1 \mathrm{~m}$ and then it increases for $D \geq 161.1 \mathrm{~m}$. This is because, for $D$ between $120.8 \mathrm{~m}$ and $161.1 \mathrm{~m}$, we tend to turn on more APs transmitting at either the highest power level or the second power level (Figure 4(a)) and thereby users will experience a higher throughput thus a lower delay. Note that all the curves tend to converge to the same point. This is expected because when $D$ increases, the cell overlap decreases and thus the optimal solution for the three cases tend to turn on the APs, to achieve a point where all the APs transmit at the highest power level and therefore the problem will be a user association problem that minimizes the sum of delays of all users.

\section{CONClusion}

In this paper, we advocate a multiobjective optimization for the double problem of power saving and user QoS satisfaction in a green wireless access network. Thus, we formulate a nonlinear optimization problem that consists in finding a tradeoff between reducing the power consumption of the network and selecting the best user association that incurs the lowest sum of data unit transmission user delay. We provide a linearization process of our problem that makes it computationally tractable for realistic scenarios. Different cases reflecting various decision preferences are studied by tuning the weights of the power and delay components of the network total cost. Compared to the most frequently deployed WLAN networks where APs transmit at a fixed transmit power level, results show that we obtain power savings up to $20 \%$. For future work, we plan to examine heuristic methods to solve the problem for dense scenarios.

\section{REFERENCES}

[1] The Benefits of Centralization in WLANs via the Cisco Unified Wireless Network, 2006.

[2] Channels and Maximum Power Settings for Cisco Aironet Lightweight Access Points, 2008.

[3] O. Eunsung and B. Krishnamachari. Energy savings through dynamic base station switching in cellular wireless access networks. In Proc. IEEE GLOBECOM, USA, 2010

[4] Andrea Goldsmith. Wireless Communications. Cambridge University Press, 2005

[5] O. J. Grodzevich and O. Romanko. Normalization and other topics in multi-objective optimization. In Workshop of the Fields MITACS Industrial Problems, Canada, 2006.

[6] M. Heusse, F. Rousseau, G. Berger-Sabbatel, and A. Duda. Performance anomaly of $802.11 \mathrm{~b}$. In Proc. IEEE INFOCOM, USA, 2003.

[7] K. Khawam, M. Ibrahim, J. Cohen, S. Lahoud, and S. Tohme. Individual vs. global radio resource management in a hybrid broadband network. In Proc. IEEE ICC, Japan, 2011.

[8] J. Lorincz, A. Capone, and M. Bogarelli. Energy savings in wireless access networks through optimized network management. In Proc. IEEE ISWPC, Italy, 2010.

[9] F. Moety, M. Ibrahim, S. Lahoud, and K. Khawam. Distributed heuristic algorithms for rat selection in wireless heterogeneous networks. In Proc. IEEE WCNC, France, 2012.

[10] F. Richter, A. J. Fehske, and G. P. Fettweis. Energy efficiency aspects of base station deployment strategies for cellular networks. In Proc. IEEE VTC Fall, USA, 2009.

[11] K. Son, H. Kim, Y. Yi, and B. Krishnamachari. Base station operation and user association mechanisms for energy-delay tradeoffs in green cellular networks. IEEE JSAC, 2011.

[12] S. Tombaz, M. Usman, and J. Zander. Energy efficiency improvements through heterogeneous networks in diverse traffic distribution scenarios. In Proc. IEEE ICST, China, 2011. 